\title{
Una aproximación al crecimiento y desarrollo económico desde los ciclos de mejora
}

\author{
JuAn AlBerto HuEso ARRABAL \\ Departamento de Economía Aplicada I (FCEYE) \\ Universidad de Sevilla \\ juanalbertohueso@us.es \\ ORCID: https://orcid.org/0000-0002-4069-2940 \\ D.O.I.: http://dx.doi.org/10.12795/JDU.2018.i01.85 \\ Pp.: 1514-1531
}

\section{Resumen}

En un determinado contexto sobre los pilares de la Economía, encontramos la cuestión relacionada con el crecimiento y desarrollo económico. Para el mejor entendimiento de la temática, se ha abordado un ciclo de mejora basado en el actual curso académico 2018-2019. Hoy día, los modelos no se centran en que el alumno tenga un aprendizaje de calidad y duradero en el tiempo. Si no que las evaluaciones, contenidos y el modo en que se incentiva al alumno están arraigados a una enseñanza estancada que no está adaptada a las necesidades que se generan con el paso del tiempo (Noguero, 2005). Una mejora centrada en la orientación de los criterios y en la aplicabilidad de los contenidos es la justificación por la que se ha realizado este ciclo de mejora. El resultado del ciclo de mejora ha sido muy positivo, incrementándose la comprensión de los contenidos por parte de los alumnos. 
Palabras clave: Introducción economía; Grado en contabilidad y finanzas; Docencia universitaria; Experimentación docente universitaria; Crecimiento y desarrollo económico

\section{Breve descripción del contexto}

El ciclo de mejora realizado se centra en la asignatura de Introducción a la Economía para el Grado de Contabilidad y Finanzas. Se trata de una asignatura obligatoria con una carga de 6 ECTS y centrada en conceptos e ideas puramente teóricos. Al ser una asignatura de primer curso, se pretende que el alumno adquiera unas herramientas y conceptos que les van a ser requeridos en futuras asignaturas.

La media de edad se sitúa entre 18 y 20 años. Esto implica que el escenario es un tanto hostil. Estos alumnos acaban de dar el salto a la universidad y cuestiones de una indole abstracta como puede ser el crecimiento y desarrollo económico, haberlo hecho "atractivo" para su aprendizaje ha sido una complicada tarea. Desde una perspectiva dinámica y entretenida se ha pretendido obtener un primer acercamiento de estos alumnos a la rama de la economía. Las empresas y el mundo que rodea a las empresas globalizadas hace que dichas cuestiones sean totalmente necesarias para la formación del alumno.

Este ciclo ha tenido como base el realizado el pasado curso académico en el que por medio de un aprendizaje colaborativo y dinámico se intentó potenciar la aplicación de la metodología. Las debilidades encontradas el pasado curso fueron las mejoras que se han implantado en este ciclo. El ciclo se realizó para diez horas a lo largo de cinco sesiones comprendidas entre el 11/10/2018 y el 25/10/2018. Cabe destacar que los resultados obtenidos fueron positivos. Los alumnos reconocieron que las actividades desarrolladas fueron dinámicas y atractivas para el aprendizaje 
por lo que ellos mismo reforzaron la efectividad de la metodología usada.

\section{Diseño previo del ciclo de mejora docente}

\section{Mapa de contenidos}

Los objetivos abordados y los contenidos perseguidos para la comprensión del crecimiento y desarrollo económico fueron los siguientes:

- Fallos del mercado

- Intervención del estado: Fallos del sector público

- Qué es el crecimiento económico

- Cómo se mide el crecimiento económico de los países

- Bases y modelos del crecimiento económico

- Internacionalización de los mercados

- Globalización de las economías

- Qué es el Desarrollo Económico

- Cómo se mide el Desarrollo Económico

- Implicaciones crecimiento y desarrollo económico

El mapa de contenidos está representado en la siguiente Figura 1 donde gráficamente puede verse como:

Jornadas de Formación e Innovación Docente del Profesorado | № 1 (2018) Esta obra se distribuye con la licencia Creative Commons 


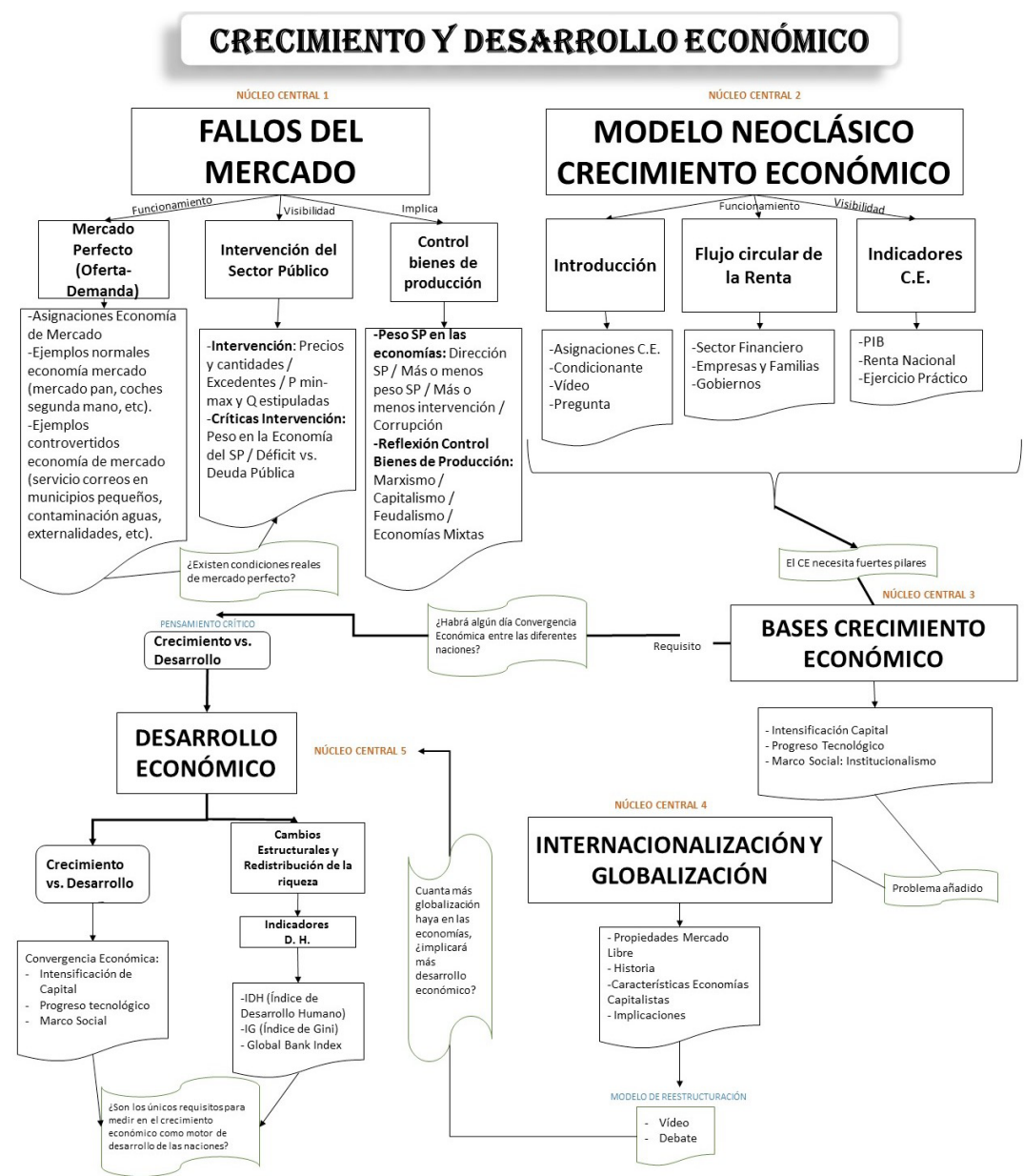

Figura 1: Mapa de contenidos

A continuación se detallan las preguntas clave de razonamiento realizadas a lo largo de las sesiones para lograr la efectividad del pensamiento guiado:

1. Crisis financiera de 2008. ¿Cambio de paradigma? ¿Qué alcance: filosófico, económico, laboral, político, ecológico, etc.?

2. ¿Creéis que el mecanismo de Oferta y Demanda es eficiente en la asignación de recursos?

3. ¿Por qué debería de intervenir el Sector Público?

4. ¿Qué debería de medir el Crecimiento Económico? 
5. ¿Por qué el Crecimiento Económico se mide por la producción y la renta?

6. ¿Hay algo más después de producir y comprar?

7. ¿Existe crecimiento sostenible, ético y responsable en las sociedades de hoy día?

8. Si existe Ceteris Paribus, ¿cómo afecta un aumento del tamaño de la población a la producción total? ¿y a la producción Per Cápita?

9. Si los países NO desarrollados crecen mucho pero su PIBp.c. es muy pequeño, ¿habrá algún día Convergencia Económica?

10. Un país subdesarrollado o en vías de desarrollo, ¿qué debería hacer para mejorar la situación? ¿Dónde debería de invertir?

11. ¿Capital físico o educación? o ¿Estarse quieto y seguir con su ritmo normal y obviar que hay otras maneras de vivir?

12. ¿Habrá algún día convergencia entre los países desarrollados y los que están en vía de desarrollo / subdesarrollo?

\section{Modelo metodológico posible y secuencia de actividades programadas}

El ciclo de mejora se ha diseñado para 10 horas. Este modelo se desarrolla a partir de las metodologías adquiridas en el curso de Profesorado de Formación e Innovación Docente en el 2017 donde se realizó el primer testeo de estas metodologías Hueso (2017). Las debilidades encontradas en esta comunicación junto con las metodologías aportadas por Finkel (2000) y Bain (2006) han servido como base para perseguir los siguientes objetivos en el actual CMD:

- Familiarizar al alumno con la temática mediante la comprensión del contexto: Para el correcto entendimiento 
de los conocimientos, se verá la aplicación real que tienen los contenidos que se desarrollan.

- Incentivar y motivar al alumno con las cuestiones desarrolladas: Mediante un proceso guiado de razonamiento, la participación activa de los alumnos y la realización de determinadas actividades hará que el alumno empiece a familiarizarse con los contenidos de la asignatura.

- Interiorizar los conceptos aplicados para que el alumno tenga argumentos reales teóricos-prácticos capaces de justificar su razonamiento: Por medio del aprendizaje cooperativo, se persigue una interacción entre el profesor y el alumno que permita el desarrollo de los contenidos desde el aprendizaje significativo, es decir, aquel que se lleva a cabo con lógica y relacionando los componentes cognitivos.

- Ejercer en ellos un pensamiento independiente: En última instancia, se persigue que los conocimientos adquiridos sean de una índole duradera en el tiempo, para que sean capaces desenvolverse de manera independiente por haber interiorizado los conocimientos. Los debates y discusiones generados a lo largo de las sesiones determinan que los alumnos hayan sido capaces de asimilar los conceptos.

Para la mejor aplicación del CMD, cabe decir que todo el trabajo que se realiza por parte del docente así como por el alumno es un trabajo realizado durante las sesiones. A grandes rasgos, las actividades se basaron en el modelo metodológico basado en el Proceso Guiado de Aprendizaje (véase Figura 2). La motivación y la captación de la temática será el elemento diferenciador para este alumnado; intentando presentar casos prácticos y actuales que puedan involucrar activamente al alumno. La argumentación, el contraste de las opiniones y la puesta en común de los criterios será la mejora esperada para el alumno. 


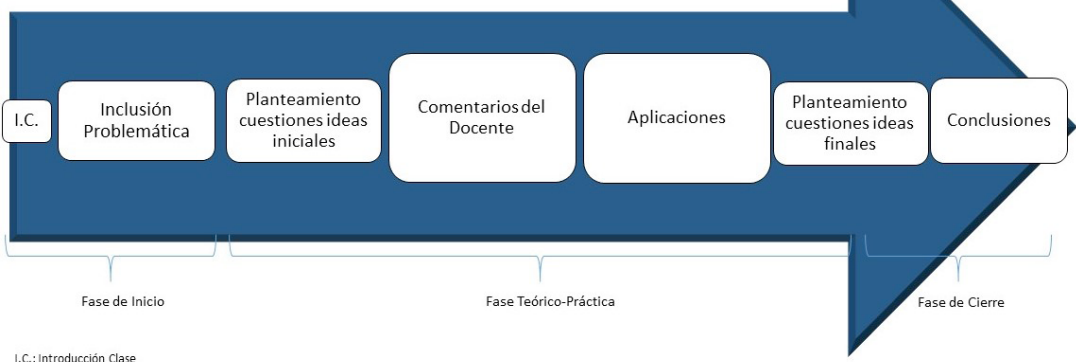

Figura 2: Modelo metodológico del Ciclo de Mejora

El modelo que hemos diseñado tiene una primera etapa llamada "Fase de Inicio" en la cual se pudo poner al alumno en una posición para el aprendizaje; comprendida por la introducción de los contenidos que iban a exponerse en clase y la problemática que iba a anticipar la sesión. Tras ello, llegó una segunda "Fase Teórico-Práctica" donde por medio de los comentarios del docente y la evaluación de la aplicación real de los contenidos se introdujo la línea de la sesión. Respecto a la "Fase de Cierre", se eligió contenido digital para poder realizar debates y con ello, pudieran contrastar las ideas iniciales.

A la hora de abordar la realización de este diseño del primer ciclo de mejora, se ha basado en el modelo Transitivo Puro de conocimiento. En los sucesivos cuadros, se muestra la secuencia detallada y temporizada de las actividades implantadas: 
Sesión 1: 11/10/2018

\begin{tabular}{|c|c|c|c|}
\hline Fase del modelo & Componentes & Secuencia actividades & T. \\
\hline $\begin{array}{l}\text { Introducción } \\
\text { Clase }\end{array}$ & 1. Saludo y llegada & $\begin{array}{l}\text { - Contacto visual para } \\
\text { implantar silencio, } \\
\text { orden en la clase y } \\
\text { establecer un clima } \\
\text { de predisposición } \\
\text { para el alumno. }\end{array}$ & $10 \mathrm{~min}$ \\
\hline $\begin{array}{l}\text { Inclusión } \\
\text { Problemática }\end{array}$ & $\begin{array}{l}\text { 2. Cuestionario ideas } \\
\text { iniciales de los } \\
\text { alumnos }\end{array}$ & 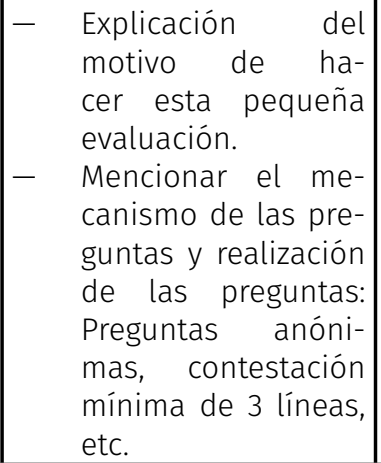 & $15 \mathrm{~min}$ \\
\hline $\begin{array}{l}\text { Comentarios } \\
\text { del Docente y } \\
\text { aplicaciones }\end{array}$ & $\begin{array}{l}\text { 3. Fallos del Mercado: } \\
\text { Condiciones Mer- } \\
\text { cado Perfecto }\end{array}$ & {$\left[\begin{array}{l}\text { - } \\
\text { llo tradeo "Mercadi- } \\
\text { Marruecos" } \\
\text { - } \\
\text { Preguntas intro- } \\
\text { ductorias inicio del } \\
\text { pensamiento crítico. }\end{array}\right.$} & $35 \mathrm{~min}$ \\
\hline $\begin{array}{l}\text { Comentarios } \\
\text { del Docente y } \\
\text { aplicaciones }\end{array}$ & & \begin{tabular}{|ll} 
- & Vídeo "Compa- \\
& ñías eléctricas en \\
España". & \\
- & P r e g u n t a s \\
razonamiento
\end{tabular} & $35 \mathrm{~min}$ \\
\hline $\begin{array}{l}\text { Planteamiento } \\
\text { cuestiones ideas } \\
\text { finales }\end{array}$ & $\begin{array}{l}\text { 4. Fallos del Mercado: } \\
\text { Intervención del } \\
\text { Sector Público }\end{array}$ & $\begin{array}{ll}\text { - } & \text { Gremio de los } \\
& \text { taxistas } \\
\text { - } & \text { Impuesto al sol en } \\
& \text { España } \\
\text { - } & \text { P r e g u n t a s } \\
\text { razonamiento } \\
\text { - Debate }\end{array}$ & $20 \mathrm{~min}$ \\
\hline Conclusiones & 5. Repaso sesión & & $5 \mathrm{~min}$ \\
\hline
\end{tabular}

Jornadas de Formación e Innovación Docente del Profesorado | № 1 (2018) Esta obra se distribuye con la licencia Creative Commons Reconocimiento-NoComercial-SinObraDerivada Internacional (CC BY-NC-ND 4.0.) 
Sesión 2: 15.10 .2018

\begin{tabular}{|c|c|c|c|}
\hline Fase del modelo & Componentes & Secuencia actividades & $\mathbf{T}$. \\
\hline \multirow[t]{2}{*}{$\begin{array}{l}\text { Introducción } \\
\text { Clase }\end{array}$} & 1. Saludo y llegada & $\begin{array}{l}\text { - Contacto visual para } \\
\text { implantar silencio, } \\
\text { orden en la clase y } \\
\text { establecer un clima } \\
\text { de predisposición } \\
\text { para el alumno. }\end{array}$ & $10 \mathrm{~min}$ \\
\hline & $\begin{array}{l}\text { 2. Repaso sesión } \\
\text { anterior }\end{array}$ & & $10 \mathrm{~min}$ \\
\hline $\begin{array}{l}\text { Inclusión Pro- } \\
\text { blemática y } \\
\text { Planteamiento } \\
\text { cuestiones ideas } \\
\text { iniciales }\end{array}$ & \multirow{3}{*}{$\begin{array}{l}\text { 3. Fallos del Mer- } \\
\text { cado: Interven- } \\
\text { ción del Sector } \\
\text { Público }\end{array}$} & $\begin{array}{l}\text { - Artículo Le Monde } \\
\text { Diplomathique: La } \\
\text { oferta y la demanda, } \\
\text { los Adán y Eva de la } \\
\text { Economía. }\end{array}$ & $20 \mathrm{~min}$ \\
\hline $\begin{array}{l}\text { Comentarios } \\
\text { del Docente y } \\
\text { aplicaciones }\end{array}$ & & $\begin{array}{l}\text { - Fallos del Mercado: } \\
\text { Ejemplos prácticos } \\
\text { y videos para cada } \\
\text { caso: gentrificación } \\
\text { en Sevilla y Barce- } \\
\text { lona, desregulación } \\
\text { start-ups, caso prefe- } \\
\text { rentes en España, sa- } \\
\text { nidad en EEUU. }\end{array}$ & $20 \mathrm{~min}$ \\
\hline $\begin{array}{l}\text { Comentarios del } \\
\text { Docente y Plan- } \\
\text { teamiento cues- } \\
\text { tiones ideas } \\
\text { finales }\end{array}$ & & $\begin{array}{ll}\text { - } & \text { Críticas intervención } \\
\text { del sector público }\end{array}$ & $30 \mathrm{~min}$ \\
\hline $\begin{array}{l}\text { Planteamiento } \\
\text { cuestiones ideas } \\
\text { finales }\end{array}$ & $\begin{array}{l}\text { 4. Control bienes } \\
\text { de producción }\end{array}$ & $\begin{array}{ll}\text { - } & \text { Preg u } \mathrm{n} \mathrm{t} \text { a } \mathrm{s} \\
& \text { razonamiento } \\
\text { - } & \text { Debate }\end{array}$ & $25 \mathrm{~min}$ \\
\hline Conclusiones & 5. Repaso sesión & & $5 \mathrm{~min}$ \\
\hline
\end{tabular}

Jornadas de Formación e Innovación Docente del Profesorado I № 1 (2018) Esta obra se distribuye con la licencia Creative Commons Reconocimiento-NoComercial-SinObraDerivada Internacional (CC BY-NC-ND 4.0.) 
Sesión 3: 18/10/2018

\begin{tabular}{|c|c|c|c|}
\hline Fase del modelo & Componentes & Secuencia actividades & T. \\
\hline \multirow[t]{2}{*}{$\begin{array}{l}\text { Introducción } \\
\text { Clase }\end{array}$} & 1. Saludo y llegada & $\begin{array}{l}\text { - Contacto visual para } \\
\text { implantar silencio, } \\
\text { orden en la clase y } \\
\text { establecer un clima } \\
\text { de predisposición } \\
\text { para el alumno. }\end{array}$ & $10 \mathrm{~min}$ \\
\hline & $\begin{array}{l}\text { 2. Repaso sesión } \\
\text { anterior }\end{array}$ & & $10 \mathrm{~min}$ \\
\hline $\begin{array}{l}\text { Inclusión Pro- } \\
\text { blemática y co- } \\
\text { mentarios del } \\
\text { docente }\end{array}$ & $\begin{array}{l}\text { 3. Introducción } \\
\text { crecimiento } \\
\text { económico: } \\
\text { Flujo circular de } \\
\text { la renta }\end{array}$ & $\begin{array}{ll}\text { - } & \text { Teoría } \\
\text { - } & \text { Gráficos }\end{array}$ & $15 \mathrm{~min}$ \\
\hline $\begin{array}{l}\text { Inclusión Pro- } \\
\text { blemática, apli- } \\
\text { caciones y } \\
\text { Planteamiento } \\
\text { cuestiones ideas } \\
\text { iniciales }\end{array}$ & $\begin{array}{l}\text { 4. Introducción } \\
\text { crecimiento } \\
\text { económico: } \\
\text { Cómo se mide } \\
\text { el Crecimiento } \\
\text { Económico }\end{array}$ & $\begin{array}{ll}\text { - } & \text { Teoría } \\
- & \text { Vistazo INE y Banco } \\
& \text { Mundial } \\
- & \text { Gráficos PIB + RN } \\
- & \text { Fallos Indicadores } \\
& \text { Económicos } \\
- & \text { Ejercicio Práctico } \\
& \text { cálculo variaciones } \\
\end{array}$ & $50 \mathrm{~min}$ \\
\hline $\begin{array}{l}\text { Comentarios del } \\
\text { Docente y Plan- } \\
\text { teamiento cues- } \\
\text { tiones ideas } \\
\quad \text { finales } \\
\end{array}$ & $\begin{array}{l}\text { 5. Crecimiento } \\
\text { Económico }\end{array}$ & $\begin{array}{ll}\text { - } & \text { Definición } \\
\text { - } & \text { Preg u n t a s } \\
& \text { razonamiento } \\
\text { - } & \text { Debate }\end{array}$ & $30 \mathrm{~min}$ \\
\hline Conclusiones & 6. Repaso sesión & & $5 \mathrm{~min}$ \\
\hline
\end{tabular}

Sesión 4: 22/10/2018

\begin{tabular}{|l|l|l|l|}
\hline Fase del modelo & Componentes & Secuencia actividades & T. \\
\hline \multirow{2}{*}{$\begin{array}{l}\text { Introducción } \\
\text { Clase }\end{array}$} & $\begin{array}{l}\text { Saludo } \\
\text { llegada }\end{array}$ & $\begin{array}{l}\text { Contacto visual para } \\
\text { implantar silencio, or- } \\
\text { den en la clase y es- } \\
\text { tablecer un clima de } \\
\text { predisposición para el } \\
\text { alumno. }\end{array}$ & $10 \mathrm{~min}$ \\
\cline { 2 - 5 } & $\begin{array}{l}\text { Repaso sesión } \\
\text { anterior }\end{array}$ & $10 \mathrm{~min}$ \\
\hline
\end{tabular}

Jornadas de Formación e Innovación Docente del Profesorado | № 1 (2018) Esta obra se distribuye con la licencia Creative Commons Reconocimiento-NoComercial-SinObraDerivada Internacional (CC BY-NC-ND 4.0.) 


\begin{tabular}{|c|c|c|c|}
\hline $\begin{array}{l}\text { Comentarios } \\
\text { del Docente y } \\
\text { aplicaciones }\end{array}$ & $\begin{array}{l}\text { 3. Bases del cre- } \\
\text { c i m i e n t o } \\
\text { Económico }\end{array}$ & $\begin{array}{ll}\text { - } & \text { Teoría } \\
\text { - } & \text { Gráficos } \\
\text { - } & \text { Video: "Programado } \\
& \text { para Caducar" } \\
\text { - } & \text { P r e g u n t a s } \\
& \text { razonamiento } \\
\text { - } & \text { Paradigma } \\
\text { - } & \text { Debate } \\
\end{array}$ & $80 \mathrm{~min}$ \\
\hline $\begin{array}{l}\text { Planteamiento } \\
\text { cuestiones } \\
\text { ideas finales }\end{array}$ & 4. Kahoot & & $15 \mathrm{~min}$ \\
\hline Conclusiones & 5. Repaso sesión & - Dudas & $5 \mathrm{~min}$ \\
\hline
\end{tabular}

Sesión 5: 25/10/2018

\begin{tabular}{|c|c|c|c|}
\hline Fase del modelo & Componentes & Secuencia actividades & $\mathbf{T}$. \\
\hline \multirow[t]{2}{*}{$\begin{array}{l}\text { Introducción } \\
\text { Clase }\end{array}$} & 1. Saludo y llegada & $\begin{array}{l}\text { - Contacto visual para } \\
\text { implantar silencio, } \\
\text { orden en la clase y } \\
\text { establecer un clima } \\
\text { de predisposición } \\
\text { para el alumno. }\end{array}$ & $10 \mathrm{~min}$ \\
\hline & $\begin{array}{l}\text { 2. Repaso sesión } \\
\text { anterior }\end{array}$ & & $10 \mathrm{~min}$ \\
\hline $\begin{array}{l}\text { Inclusión } \\
\text { Problemática }\end{array}$ & $\begin{array}{l}\text { 3. Interna cio- } \\
\text { nalización y } \\
\text { globalización }\end{array}$ & 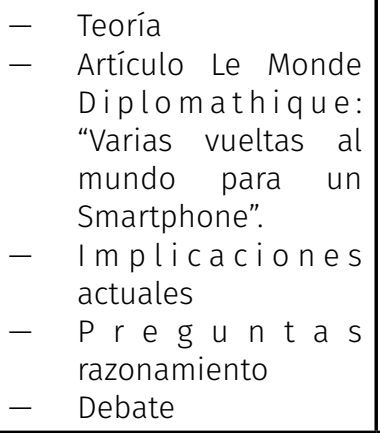 & $20 \mathrm{~min}$ \\
\hline $\begin{array}{l}\text { Comentarios } \\
\text { del docente y } \\
\text { aplicaciones }\end{array}$ & $\begin{array}{l}\text { 4. D e sarrollo } \\
\text { Económico }\end{array}$ & $\begin{array}{ll}\text { - } & \text { Teoría } \\
\text { - } & \text { Video } \\
\text { - } & \text { Preguntas proceso } \\
& \text { razonamiento } \\
\text { - } & \text { Paradigma escuelas } \\
& \text { de pensamiento } \\
\text { - } & \text { Debate }\end{array}$ & $45 \mathrm{~min}$ \\
\hline
\end{tabular}

Jornadas de Formación e Innovación Docente del Profesorado I № 1 (2018) Esta obra se distribuye con la licencia Creative Commons Reconocimiento-NoComercial-SinObraDerivada Internacional (CC BY-NC-ND 4.0.) 


\begin{tabular}{|l|ll|l|l|}
\hline Conclusiones & 5. Repaso sesión & - Dudas & $5 \mathrm{~min}$ \\
\hline Aplicaciones & 6. Kahoot & & $15 \mathrm{~min}$ \\
\hline & & & $-\begin{array}{l}\text { Explicación del mo- } \\
\text { tivo de hacer esta } \\
\text { pequeña evaluación. }\end{array}$ & $15 \mathrm{~min}$ \\
Planteamiento \\
cuestiones ideas \\
iniciales
\end{tabular}

\section{Cuestionario inicial-final para hacer un seguimiento de la evolución de los estudiantes en relación con los problemas claves}

A modo de preguntas introducidas, al comienzo de la primera sesión donde se ha implantado el CMD, el pasado jueves 11 de octubre de 2018 fueron entregadas las cuestiones para evaluar el conocimiento de las ideas iniciales de los alumnos. La intención residía en que al final de las cinco sesiones se volviera a hacer entrega de las mismas o similares preguntas, para ver la evolución por parte de ellos respecto a los conceptos adquiridos y pudieran ser contrastados con sus conocimientos iniciales.

Los cuestionarios para evaluar las ideas iniciales y finales de los alumnos consistieron en 3 preguntas cortas. El cuestionario fue totalmente anónimo aunque se pidió que escribiesen un código identificativo para ellos mismos, dónde sólo ellos pudieran ser identificados y así de esta manera, para poder evaluar de manera anónima la evolución puntual de cada alumno.

Las cuestiones iniciales fueron:

i. ¿Debería el sector público corregir los fallos del mercado? (Excedentes, pérdidas de eficiencia, etc.) 
ii. ¿En qué medida afectan los Fallos del Mercado al crecimiento económico de los países?

iii. ¿Cómo debería de medirse el crecimiento económico de los países?

Las cuestiones finales consistieron en:

i. ¿Debería el sector público corregir los fallos del mercado?

ii. ¿En qué medida afectan los Fallos del Mercado al crecimiento económico de los países?

iii. ¿Cómo debería de medirse el crecimiento económico de los países?

\section{Aplicación del ciclo de mejora docente}

\section{Relato resumido de las sesiones}

Los alumnos valoraron positivamente el haber efectuado un ciclo de mejora para los contenidos elegidos. valoraron el uso de medios audiovisuales así como haber impartido dinamismo en el trascurso de las sesiones. Los debates, las discusiones generadas y el haber visto al alumnado predispuesto para intervenir fueron de los avances más notable que encontré al haber aplicado este ciclo de mejora.

Cabe decir que se pueden apreciar dos tipos de alumnos: los más activos y los sujetos pasivos. Tras varias preguntas encontré que muchos de los alumnos y alumnas que intervenían eran siempre los mismos Al ser alumnos de primer curso y al lanzar preguntas con una abierta respuesta, hay que intentar "obligar" al alumno a que participe. En caso contrario, el esfuerzo necesario para guiar la sesión es mayor cuando no todo el mundo está involucrado de la misma manera. El docente en este punto está 
en la obligación de anticiparse a que no ocurra esto ya que luego motivar al alumno y obligarle a que participe es más complicado. Hay que intentar hacerles partícipes desde el momento uno para que estén en el clima necesario que requiere la sesión.

No obstante, a modo de refuerzo positivo, personal y de mejora, la participación del alumno en los debates me ha tranquilizado. El esfuerzo por querer participar en los debates y lidiar con cuestiones muy amplias y subjetivas lo valoré como muy importante. El ejercicio practicado por ellos de trasladar nuevas ideas y verbalizarlas, y materializarlas en palabras sostenidas por sus argumentos, lo valoré como un ejercicio muy positivo para ellos.

\section{Evaluación del aprendizaje de los estudiantes. Escaleras de Aprendizaje.}

La manera en la que se ha llevado a cabo la evaluación del aprendizaje del alumno ha consistido principalmente en la evolución de las ideas que ellos han tenido. El contraste de la información sobre las ideas iniciales y finales de los alumnos ha sido la única evaluación real del aprendizaje que hemos tenido en cuenta. Sin embargo, este aprendizaje es muy relativo porque no hay mucho espacio de tiempo para el asentamiento de los contenidos aprendidos en clase. Cuestiones como la capacidad oratoria, iniciativa a la hora de participar, la capacidad del directo y enlace de ideas surgidas durante el debate, entre otras capacidades, deberían de tenerse en cuenta, pero en nuestro caso no se ha podido hacer.

A la hora de elaborar las escaleras de aprendizaje para medir las ideas y aprendizaje de los alumnos, se hizo una clasificación para su mejor entendimiento. En primer lugar, se tuvieron en cuenta las respuestas que no tenían nada que ver con lo que se preguntaba y se clasificó como 
"Conceptos no relacionados". Por otra parte, la clasificación "Respuestas intuitivas sin fundamento" se incluían respuestas que no tenían ninguna idea que justificaran la contestación del alumno. Por otro lado, se clasificaron las "Respuestas intuitivas con fundamento" dónde el alumno contestó de manera intuitiva pero argumentando su respuesta. Otra clasificación fue la de "Enlace de ideas y ejemplos ilustrativos" dónde el alumno contestó a la pregunta argumentando y enlazó conceptos con la ayuda de ejemplos prácticos para la mejor comprensión. Por último, la clasificación "Conocimiento teórico con fundamento en la respuesta" englobó a las respuestas que estaban muy bien elaboradas y fundamentadas con conocimiento teórico.

En la siguiente Figura 3 se puede evaluar el grado de las respuestas que tuvieron los alumnos en el cuestionario de las ideas iniciales. Era de esperar que al haber preguntado cuestiones de una índole más abstracta, a términos generales no contestaran de la manera en la que se esperaba. Cabe destacar que el 33\% de los alumnos no tenía prácticamente alguna idea de lo que se estaba preguntando. Por el contrario, tan sólo un 7\% contestó acorde a lo que se esperaba. El resto de respuestas se dividieron en contestaciones intuitivas, donde a veces con ejemplos prácticos, orientaban las respuestas pero en su gran mayoría carecían de alguna idea formulada.

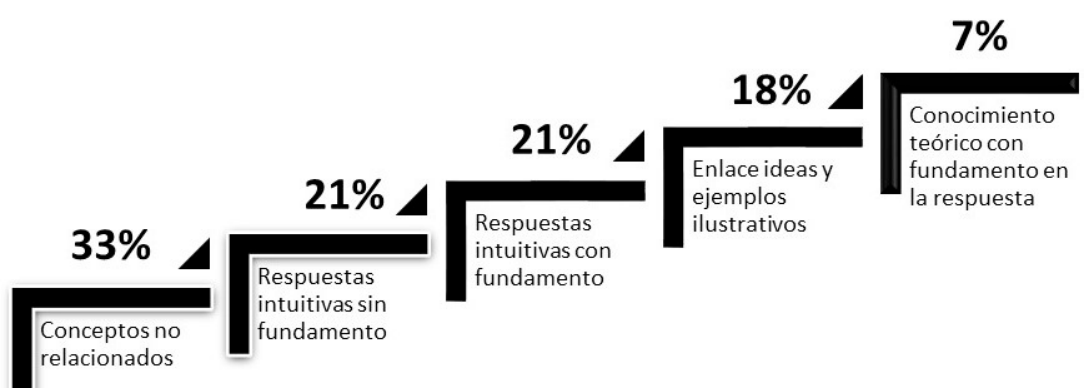

Figura 3: Evaluación de las ideas iniciales $(n=39)$

Jornadas de Formación e Innovación Docente del Profesorado | № 1 (2018) Esta obra se distribuye con la licencia Creative Commons Reconocimiento-NoComercial-SinObraDerivada Internacional (CC BY-NC-ND 4.0.) 


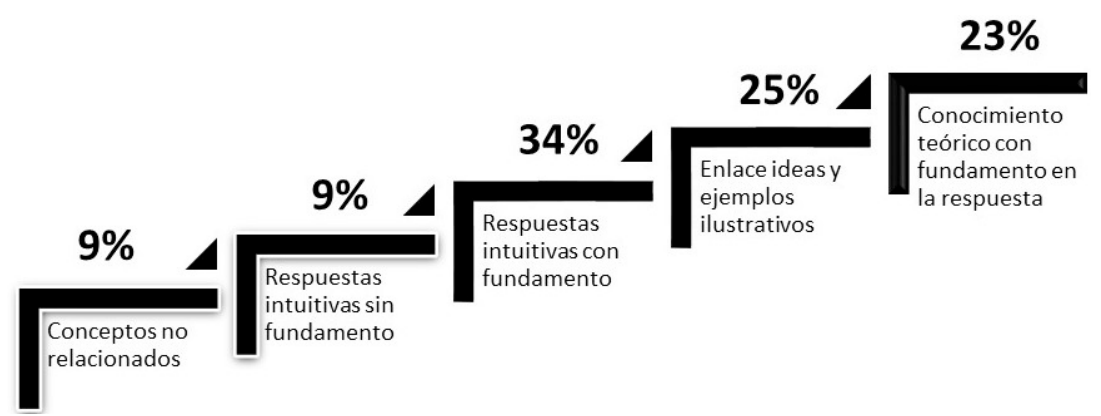

Figure 4: Evaluación de las ideas finales $(n=31)$

En la Figura 4 se muestran las contestaciones que los alumnos tuvieron a la hora de evaluar las ideas finales de los contenidos. El mayor logro que se puede destacar es que después de la aplicación del ciclo de mejora, tan sólo el $9 \%$ de los alumnos siguieron teniendo alguna o poca idea sobre los contenidos en los que se basó el ciclo. Podría argumentarse que a excepción de 2 individuos, el resto fueron alumnos que no realizaron el primer cuestionario. Al haber pedido que los alumnos hicieran marcas anónimas e individuales para hacer un seguimiento de los cuestionarios, los resultados que se clasificaron como "Conceptos no relacionados" fueron alumnos que no han asistido regularmente a las sesiones, al menos a las sesiones cuando se repartieron los cuestionarios. El 23\% de los alumnos respondieron de la manera esperada, argumentando sus respuestas y mostrando el entendimiento y dominio de los conceptos. Cabe destacar que entorno al 60\%, los alumnos siguieron contestando de manera intuitiva aunque presentaron argumentos que justificaban su respuesta. Este hecho presenta una mejora y otro punto positivo a tener en cuenta por la aplicación del ciclo de mejora.

A rasgos generales, se observa una mejora por la aplicación del CMD por los resultados que se han obtenido. Sin embargo, no todo es sumamente positivo porque sigue habiendo un alto porcentaje de alumnos (en torno al 45\%) que no pudieron hacer frente a sus respuestas justificando 
con elementos teóricos o ejemplos prácticos usados durante las sesiones que justificaran sus ideas. Según (Vigotsky, 2015), la justificación que existe en este punto se debe a que hay determinadas situaciones en las que el alumno tiene dificultades para elaborar respuestas adecuadas o aún no tiene la suficiente capacidad para elaborarlas. Más aun teniendo en cuenta que son alumnos de primer año en una asignatura un tanto alejada de la contabilidad y las finanzas.

\section{Evaluación del ciclo de mejora docente}

A modo de resumen, a continuación se muestran los cambios más notables para establecerlos de cara a un próximo ciclo de mejora:

- Establecer nuevos criterios de evaluación donde se evalúen más competencias del alumno.

- Seguir trabajando en mejorar los mapas de contenidos para enlazar de una mejor manera los contenidos a desarrollar con los problemas clave. Aún existen algunas debilidades que se podrían mejorar.

- Limitación en el tiempo. Habría que dar más tiempo para asentar las ideas y los contenidos desarrollados para que los alumnos puedan interiorizarlos de mejor manera.

- Exigir continuidad en los alumnos. No todos los alumnos asistieron a la totalidad de las sesiones. La efectividad del ciclo no se puede extrapolar al cien por cien de los alumnos.

- Coordinar los planes de organización de la asignatura con la metodología que usamos en los CMD y hacerlas reales en los grupos donde se aplican los ciclos.

Por otra parte, a niveles generales vamos a seguir manteniendo las siguientes cuestiones ya que la aplicación de ellas sigue siendo positiva y favorable: 
- Al realizar debates y estimular el pensamiento crítico, los alumnos se involucraban en las sesiones denotando interés y curiosidad por la materia.

- Plantear problemas reales para llevar a cabo la explicación de los contenidos despierta en los alumnos interés por la materia.

- Sinergias positivas por las intervenciones de ellos.

- Uso de medios audiovisuales así como nuevas tecnologías para estimular a los alumnos.

- Realización del mapa de contenidos y alta planificación de las actividades por la secuencia de las actividades como manera de facilitar las transiciones en clase.

\section{Bibliografía}

Bain, K. (2006). Lo que hacen los mejores profesores de universidad. Valencia: Universitat de València.

Finkel, D. L. (2000). Teaching with your mouth shut. Centers for Teaching and Technology - Book Library. 41.

Hueso, J. A. (2017). Estudio del crecimiento económico desde el aprendizaje participativo. IV Jornadas de Formación e Innovación Docente. Sevilla: ICE Universidad de Sevilla, 1007-1017.

Noguero, F. L. (2005). Metodología participativa en la enseñanza universitaria (Vol. 9). Ciudad: Narcea Ediciones.

Vigotsky, L. (2015). Interacción entre aprendizaje y desarrollo. España: Crítica-Grijalbo.

Jornadas de Formación e Innovación Docente del Profesorado | № 1 (2018) Esta obra se distribuye con la licencia Creative Commons 